\title{
Empirically Supported Treatments and General Therapy Guidelines for Non-Suicidal Self-Injury
}

\author{
Jennifer J. Muehlenkamp
}

\begin{abstract}
Mental health counselors are facing increased demand to treat both adolescents and adults who present with repetitive non-suicidal self-injurious behaviors, yet there are few empirically supported treatments or general treatment guidelines available. I will review the research on problem-solving and dialectical behavior therapy, two cognitive-behavioral treatments that have the most empirical support for reducing self-injurious behavior. I conclude by providing specific treatment recommendations drawn from the literature that can be of use to mental health counselors working with individuals who self-injure.
\end{abstract}

There is growing interest within the field to find effective outpatient treatment strategies for reducing non-suicidal self-injurious behaviors (NSSI). Self-injurious behavior, for the purpose of this paper, refers to acts that damage body tissue (e.g., cutting, burning) and occur without suicidal intent. Researchers are documenting increases in the prevalence of self-injurious behavior in adult populations (Hawton et al., 1997), and it is believed a similar increase is occurring within adolescent groups. Prevalence rates of NSSI have ranged from 4.3 to $35 \%$ (Briere \& Gil, 1998; Gratz, 2001), and Favazza (1998) estimated that anywhere from 400 to 1,400 per 100,000 persons engage in NSSI behaviors per year. Selfinjury has typically been identified as a behavior resistant to treatment efforts (Zila \& Kiselica, 2001), and presents many challenges to a therapist since acts of NSSI can lead to severe injuries and accidental death. In addition, individuals who engage in non-suicidal self-injury are at heightened risk for suicide, further complicating the treatment. The standard treatment approach has been hospitalization, but this is an expensive option that has not reliably demonstrated effectiveness (Linehan, 2000)

Please direct correspondence regarding this manuscript to Jennifer J. Muehlenkamp, Ph.D., Department of Psychology, PO BOX 8380, University of North Dakota, Grand Forks, ND 58202. E-mail: Jennifer.muehlenkamp@und.nodak.edu. 
for suicidal or non-suicidal self-injury. Additionally, individuals engaged in non-suicidal self-injury are unlikely to be admitted because they do not express intent to die. Consequently, hospitalization is used less frequently and many mental health counselors are left trying to treat this difficult behavior on an outpatient basis with little guidance regarding the best treatment approach.

Unfortunately, there is little empirical data offering treatment guidelines for NSSI. Few large-scale treatment studies include self-injuring or suicidal individuals because of ethical and legal risks (Linehan, 2000). Even fewer studies specifically target NSSI behaviors as the main focus for treatment (MacLeod et al., 1992; Miller \& Glinski, 2000); however, NSSI is sometimes included as a target behavior along with suicide ideation and attempts in the treatment studies that do exist, so these studies can provide some guidance.

Given that non-suicidal self-injury is primarily conceptualized as a tool for emotion regulation (Linehan, 1993; Nock \& Prinstein, 2004) maintained through positive and negative reinforcements, treatments utilizing cognitive-behavioral strategies show the greatest promise for successfully reducing the behavior. Cognitive-behavioral interventions have demonstrated effectiveness at reducing repetitive suicidal behaviors (Evans, 2000), implying potential success for the treatment to generalize to nonsuicidal self-injury. My goal for this paper is to highlight studies of interventions that show some success in treating NSSI, identify what may be the effective mechanisms of change in these studies, and conclude with a set of recommendations for how to approach the treatment of this behavior. Due to the limited number of treatment studies specific to non-suicidal self-injury, my review of empirically supported treatments will at times include studies that examined changes in both non-suicidal selfinjury and suicidal behavior.

\section{COGNITIVE BEHAVIORAL THERAPIES FOR NON-SUICIDAL SELF-INJURIOUS BEHAVIOR}

In reviewing the literature, only two types of treatments falling within the cognitive-behavioral domain that focus specifically on NSSI were identified: Problem-Solving Therapy (PST; D'Zurilla \& Goldfried, 1971) and Dialectical Behavior Therapy (DBT; Linehan, 1993). These treatments share common features such as being time-limited, structured therapies with an emphasis on immediately targeting NSSI and remedying skill deficits. Each treatment's effectiveness with reducing NSSI will be reviewed and where possible, suggestions of potential mechanisms of change will be identified. 


\section{Problem-Solving Therapy (PST)}

The major assumption underlying the use of PST is that dysfunctional coping behaviors result from a cognitive or behavioral breakdown in the problem-solving process (D'Zurilla \& Nezu, 2001). The goal of therapy is to help clients identify and resolve the problems they encounter in their lives, as well as to teach clients general coping and problem-solving skills that they can utilize in the future to deal more effectively with the problems they encounter. This is usually done by teaching the different steps in problem solving including problem identification and goal setting (often by utilizing a behavioral analysis of the problem), brainstorming and assessing potential solutions, selecting and implementing a solution, and evaluating the success of the chosen solution. Teaching these steps is viewed as important to reducing NSSI because researchers have consistently found that individuals who engage in self-injury often exhibit poor problem-solving skills (Evans, Williams, O'Loughlin, \& Howells, 1992; Pollock \& Williams, 1998; Speckens \& Hawton, 2005) and tend to have rigid thinking styles (Kernberg, 1994). As in many other treatments, PST also stresses the importance of forming a strong therapeutic relationship with the client so that the teaching and practice of skills is a collaborative process.

Research on the efficacy of PST in reducing self-injurious behaviors has found mixed support, making it difficult to draw specific conclusions. Early studies examined the effectiveness of PST compared to crisis-based social work interventions or treatment as usual. Some of the early studies found that PST was effective in significantly reducing acts of self-poisoning as well as reducing suicidal ideation compared to crisis-interventions and treatment as usual (Gibbons, Butler, Urwin, \& Gibbons, 1978; Patsiokas \& Clum, 1985). However studies by Hawton et al. (1987) and Liberman and Eckman (1981) failed to find evidence that PST was more effective than treatments as usual. All these studies were limited by small sample sizes, exclusion of individuals deemed to be at high risk for suicide, and confounding access to social work/case management services in addition to psychotherapy. More recent studies of PST have demonstrated short-term success at reducing suicidal behaviors compared to treatment as usual (e.g., McLeavey, Daly, Ludgate, \& Murray, 1994; Lerner \& Clum, 1990), but the differential improvements have not been maintained at long-term follow-up points as long as 24 months (e.g., Rudd et al., 1996; Salkovskis, Atha, \& Storer, 1990). These studies often included NSSI in their definition of suicidal behavior, making it difficult to determine whether PST has a unique treatment effect on just NSSI.

In a meta-analysis of 20 studies that used randomized clinical trials for assessing the efficacy of different treatments for "parasuicide" (including 
NSSI and suicide attempts), Hawton and colleagues (1998) found that many of the types of treatment reviewed in the meta-analysis (e.g., intensive therapy, in-patient therapy, medication) either failed to produce reductions in self-injurious behaviors, or failed to demonstrate significant reductions of the behavior compared to controls. Problem-solving therapy appeared to produce the greatest reductions in parasuicide among participants compared to standard care controls; however, the reductions found were not statistically significant. The authors subsequently concluded that there is no consensus as to what treatment strategies are best, but of the studies reviewed, problem-solving therapy showed the most promise. A follow-up meta-analysis of problem-solving therapy used to treat "deliberate self-harm" (greater emphasis on NSSI) found that PST significantly reduced comorbid depressive symptoms, hopelessness, and problem levels among participants, however results regarding PST effectiveness on reducing acts of NSSI were inconclusive (Townsend et. al., 2001). Possible reasons for the inconclusive results include the small sample of studies (only 6) and the small participant samples within each study. Furthermore, due to the difference in outcome variables measured, most of the analyses included only 3 or 4 of the original 6 studies. Therefore, it seems premature to come to any strong conclusions regarding the overall effectiveness of PST for NSSI from these meta-analyses.

A handful of studies have emerged in the past few years suggesting some potential for PST to significantly reduce NSSI. The PSTs that show greater promise for long-term efficacy in reducing NSSI are those that incorporate additional cognitive, interpersonal, or behavioral elements into the standard problem-solving protocol, suggesting that a comprehensive approach may be best. In 1999, Evans et al. used a manualassisted cognitive-behavioral therapy (MACT) to treat 34 individuals with repetitive acts of self-injurious behavior. MACT treatment is designed to be a short-term problem-solving and cognitive-behavioral intervention of 6 sessions, teaching clients skills to manage emotions and negative thinking. Of the 34 individuals in this study, 18 were randomly assigned to MACT and 16 were assigned to treatment as usual. Results indicated that the MACT group had lower rates of self-injury as well as a longer time delay between self-injurious acts compared to controls, but the difference in time delay was not statistically significant.

The effectiveness of MACT in reducing self-injurious behaviors was further tested in a multi-site, randomized clinical trial comparing MACT to treatment-as-usual for 480 clients. Tyrer et al. (2003) reported that clients who received MACT reported significantly fewer self-injurious episodes at 6 and 12 month assessment points. At the 12-month assessment point, the MACT group had a non-significantly smaller percentage 
(39\%) of individuals reporting repeated self-injury relative to the treatment as usual group (46\%). Additionally, there was a longer time delay to repeat acts of self-injury in the MACT group (222 days) compared to controls (169 days), but this difference was not significant. These findings appear to suggest that training in problem-solving may increase the effectiveness of standard treatments; however, neither of the studies addresses what aspects of MACT appear to be efficacious in reducing NSSI. The short duration of the treatment may account for the lack of significant findings in delaying repeated acts of NSSI, particularly for individuals with a long history of NSSI or those with Axis II pathology such as borderline personality disorder (Tryer et al., 2004). These individuals may require a longer treatment period than the brief MACT model to fully eradicate acts of self-injury. Overall, the results of MACT are promising, but additional research is needed to ensure its long-term effectiveness as well as to identify what the potential effective components are.

Also worth mentioning are two studies that have utilized a cognitivebehavioral problem-solving approach in the treatment of NSSI. Crowe and Bunclark (2000) evaluated an intensive multi-dimensional inpatient treatment that incorporated problem-solving skills training, cognitive restructuring, psychotropic medication, dynamic processing, and both group and family therapy. Over the four-year time period of this study, Crowe and Bunclark treated 58 self-injuring clients. Thirty-two of the participants significantly decreased their acts of self-injury, while 23 stayed the same, and 3 worsened. Although limited by sample size and a lack of a comparison group, this study suggests that comprehensive treatment approaches utilizing PST along with other skills can produce some success. In a sample of Indian patients, Raj, Kumaraiah, and Bhide (2001) employed a cognitive-behavioral approach that emphasized problemsolving. Participants were randomly assigned to either the CBT-PST group or treatment as usual. The CBT-PST group showed a significant decrease in suicidal ideation, hopelessness, and depressive symptoms. The repetition rate for self-injurious behavior was $5 \%$. Data for the self-injury rates in the control group were not reported, so conclusions specific to reductions in self-injury cannot be made, but this study provides additional support for the potential effectiveness of PST to reduce NSSI.

Overall, the research regarding the effectiveness of PST is inconclusive. The limited number of studies is the greatest obstacle in determining the contribution PST may make to reducing NSSI, although the current research indicates that it has some therapeutic effect. It is unclear from the research whether the effective ingredient is the problem-solving training or another factor within the treatment. Future studies will need to explore this question. However, it seems appropriate to conclude that 
PST show promise for effectively reducing NSSI especially when combined with other CBT skills.

\section{Dialectical Behavior Therapy (DBT)}

Dialectical behavior therapy was developed by Linehan (1993) to treat individuals with borderline personality disorder (BPD), of which NSSI behavior is a prominent symptom. DBT is based upon a conglomeration of Zen Buddhism, cognitive-behavioral interventions, problem-solving, and skills training. The core dialectical principle underlying DBT is a balance between encouraging the client to change and accept him or herself simultaneously. One of the primary treatment goals of DBT is to reduce NSSI (and suicidal) behaviors by helping clients develop new coping skill sets, addressing motivational obstacles during treatment, and promoting skill generalization outside the therapy setting (Ivanhoff, Linehan, \& Brown, 2001; Robins \& Chapman, 2004). Mastering these tasks is accomplished by utilizing a hierarchical stage model that provides a guiding structure for the therapy within a number of treatment modalities, including individual therapy, group skills training, phone coaching, and supervision/consultation for the counselor.

The pre-treatment stage involves orienting the client to the therapy and obtaining a commitment agreement for therapy. Stage one focuses specifically on reducing NSSI or suicidal behavior and maintaining therapy compliance, as well as reducing distress associated with Axis I disorders. Once the self-destructive behaviors are under control, stage two addresses ways of processing and dealing with traumatic experiences and invalidating environments. Stage three emphasizes developing and maintaining self-respect while synthesizing the skills learned (Ivanhoff et. al., 2001). To address stage one targets of reducing self-injurious behaviors, DBT strategies include the following: (a) validation of the client's experiences; (b) problem-solving techniques, including behavioral analyses of the self-injurious or suicidal behavior along with teaching of adaptive coping behaviors; (c) behavioral skills training in mindfulness, emotion regulation, interpersonal effectiveness, and distress tolerance; and (d) contingency management strategies (see Linehan, 1993).

Given that DBT addresses both NSSI and suicidal behaviors, many of the studies on DBT have grouped the two behaviors together, referred to as "parasuicide." The blending of these two behaviors into one construct is a limitation to the existing research. To be comprehensive, the following review of studies on the effectiveness of DBT will refer to both behaviors as self-harm. Where possible, results pertaining specifically to NSSI will be highlighted.

To date there have been four randomized clinical trials evaluating the 
efficacy of DBT as well as a handful of less rigorous treatment studies. Of the studies conducted, many show that DBT is successful in reducing selfharm in individuals with borderline personality disorder. Each of the randomized clinical trials have demonstrated significant reductions in selfharm behavior for individuals in DBT compared to treatment-as-usual controls up to 6-months post-treatment (Koons et al., 2001; Linehan, Armstrong, Suarez, Allmon, \& Heard, 1991; Linehan et al., 2002; Verheul et al., 2003). The significant differences between treatment groups have not been sustained beyond 12-months post-treatment (Linehan, Heard, \& Armstrong, 1993; Verheul et al., 2003), although this may be caused by the low rates of parasuicide found in both treatment groups at follow-up (Scheel, 2000).

In a study comparing the effectiveness of DBT to non-behaviorally oriented treatment by experts, Linehan, Comtois, et al. (2002) reported that DBT was more effective in reducing suicide attempts, but both treatments performed equally well in significantly reducing NSSI. In a sample of opioid dependent women, Linehan, Dimeff, et al. (2002) found that DBT was no more effective in reducing parasuicidal acts than a validation plus 12 step treatment. The lack of significant reductions in parasuicide may have been due to the low base rate of the behavior during the study period ( $17.4 \%$ of subjects engaged in a parasuicidal act). These findings suggest that although DBT is effective at reducing a range of self-harming behaviors, it may not contain uniquely efficacious elements to reduce NSSI.

In addition to randomized trials, a number of other studies have provided support for DBT's effectiveness at reducing self-harm. In a preliminary review of the effectiveness of standard DBT, Shearin and Linehan (1994) reported that DBT was superior to treatment as usual in reducing both the number and medical severity of self-harm acts across two studies with a total of 44 participants. Elwood et al (2002) implemented a standard DBT program in a community mental health clinic and found that after one year all clients in DBT had significantly reduced their self-harm; however, there was no comparison group and follow-up data were not obtained. In a case study of an 18 year-old female with a two-year history of NSSI, Bauserman (1998) found that a year-and-half long treatment with DBT effectively eliminated the NSSI and led to improvements in problem-solving skills, coping, and interpersonal relationships. Low, Jones, Duggan, Power, and MacLeod (2001) reported that their use of DBT with 10 self-harming females diagnosed with BPD produced significant reductions in self-harm, and this reduction was maintained at 6month follow-up. In addition, their clients experienced significant improvements in their coping beliefs, depressive symptoms, suicide ideation, and impulsiveness. Similar results were reported by Turner 
(2000), who found that clients in a DBT-oriented therapy had significantly fewer acts of NSSI, reduced suicide attempts, and improvements on other psychosocial variables such as depression. The results from these studies suggest that DBT is associated with significant reductions in NSSI among participants, but it is unclear what element of the treatment contributes to the improvement.

DBT has also been adapted for use in both inpatient and partial hospitalization programs and has demonstrated some success in reducing incidents of NSSI within these settings. Simpson et al. (1998) reported anecdotal evidence that their adaptation of DBT for use within a partial hospitalization program has been successful in reducing self-harm behaviors in women with BPD. Drawing conclusions from this study is limited in that the information was purely descriptive and the authors used a naturalistic comparison to rates of self-harm in their clients prior to implementing DBT. Within a sample of 130 inpatients, Barley et al. (1993) found that their inpatient DBT program led to significant reductions in rates of parasuicide relative to the rates prior to the implementation of DBT. They also compared their rates of parasuicide during their DBT implementation to rates of parasuicide on another psychiatric unit (no random assignment to units) finding that the DBT unit had significantly fewer acts, further supporting the efficacy of their inpatient DBT treatment.

Bohus and colleagues (2000) adapted standard DBT into a 3-month inpatient program and evaluated its effectiveness among 24 females with BPD. Data were collected at admission and 1-month post-discharge. Significant reductions in the number of self-injury acts as well as other measures of psychopathology were noted. However, there was no control group to ensure these effects were specific to DBT. A follow-up study by Bohus and colleagues (2004) compared their inpatient DBT to a wait list/treatment as usual group ( $n=31$ vs. $n=19)$. Participants were assessed four weeks after discharge (4 months after intake) and results showed that participants in the DBT program had significantly fewer acts of NSSI than those in the wait-list group ( $31 \%$ vs. $62 \%$ ). While these results are promising and in favor of DBT, one study found that an inpatient DBToriented group failed to significantly reduce acts of parasuicide compared to an alternative therapeutic approach (Springer, Lohr, Buchtel, \& Silk, 1996). However, methodological concerns and potential negative treatment elements (i.e., increased attention to parasuicide in the DBT group) may have led to the lack of significant findings.

Collectively, the studies reviewed suggest that DBT is effective in reducing NSSI, particularly among individuals with BPD. It is less clear whether the results will generalize beyond individuals with BPD since 
few studies have addressed this question. Still, the consensus within the literature is that DBT is associated with improvements in NSSI (e.g., Scheel, 2000). More research is needed to determine if treatment effects are maintained over time, what would help clients maintain their progress over time, as well as to determine what component(s) of DBT contribute to improvements.

In addition to Linehan's work with adults using DBT, it is important to note that Miller (1999) has adapted DBT for use as a 12-week intervention with self-harming adolescents. The adolescent version of DBT has shown some promise for effectively reducing NSSI and suicidal behaviors within adolescent samples (Miller et al., 2000; Rathus \& Miller, 2002). Others have also modified Linehan's DBT for adolescent populations and report that it appears to be effective in reducing self-harm and improving psychosocial adjustment in both inpatient and outpatient settings (Katz, Cox, Gunasekara, \& Miller, 2004). These adaptations are important because there is very little research regarding treatment efficacy with self-injuring adolescents, and research is suggesting that NSSI behavior is increasing within this group (Derouin \& Bravender, 2004).

As with problem-solving therapy, there is a lack of research identifying what elements of DBT contribute to its efficacy. However, in a study examining the effectiveness of DBT with a sample of 4 self-harming women with BPD, Shearin and Linehan (1992) found that mindfulness and dialectical techniques were most effective in reducing self-harm behavior. Similar findings were reported by Miller, Wyman, et al. (2000) for adolescents. Additionally, in Shearin and Linehan's (1992) study, weekly incidents of NSSI significantly decreased when therapists were rated as being nurturing, instructing, and providing autonomy to the client during the previous week of therapy. These findings were recently replicated by Perseius et al. (2003) who found that both clients and therapists identified the perceived effective treatment ingredients as being understanding, respect, and validation within the relationship along with acquisition of cognitive-behavioral skills. Turner (2000) also reported that client's ratings of the therapeutic alliance were significantly predictive of improvements. These studies suggest that an empathic and collaborative client-therapist alliance is a key component to the success of DBT, as are skills that enhance client's awareness of self and coping repertoires. The results are limited by the extremely small sample sizes in all the studies (n $=4$ to 24), the lack of comparison or control groups, and that all data were self-report so were subject to perception biases. Still, the findings offer preliminary suggestions as to what may be some of the effective components of DBT.

Based on the existing literature, it appears that DBT is a useful thera- 
peutic approach for treating NSSI behaviors, although one must be cautious about making generalizations from the current studies. Most of the research has focused specifically on suicidal behavior or the combination of suicidal and NSSI as one variable, limiting generalizations to NSSI alone. Studies that separated NSSI and suicidal behavior found that DBT was effective in reducing NSSI but the results were mixed regarding whether DBT was significantly better than treatment as usual. At best, the data regarding the efficacy of DBT for treating NSSI is strongly promising, but still inconclusive. Research with DBT must begin to assess its efficacy for treating NSSI separately from suicidal behavior as well as begin to empirically establish what components of DBT produce reductions in NSSI.

\section{SUMMARY AND EVALUATION OF PST AND DBT INTERVENTIONS FOR NSSI}

Based upon the research reviewed, it is likely safe to tentatively conclude that PST and DBT are effective approaches to treating NSSI behaviors. However, it is important to note that while these treatments show some success, few well conducted empirical studies specific to NSSI exist, and they rarely included randomized controlled trials. Results from the controlled studies reviewed are mixed, indicating that there is still much to be learned regarding the effective treatment of NSSI. Still, the two types of treatments reviewed have a number of strengths and can offer strategies to draw upon when entering into therapy with an individual who is engaging in NSSI.

First and foremost is the emphasis on the development of a strong, empathic, and collaborative working alliance. Findings that the therapeutic alliance was as predictive of improvements as the treatment itself (Perseius et al., 2003) suggest that without a strong therapeutic relationship the treatment is less likely to be successful. Other strengths are that both DBT and PST are time-limited, structured therapies. Providing structure to the therapy can increase adherence to treatment goals and may increase the efficiency of achieving them. The structure may also promote development of short-term goals so that the client can experience early success, and this can build commitment to the therapy. Having a time frame may help motivate clients because they are aware of an end and hope is generated that their misery will diminish. Both treatments emphasize targeting the NSSI as a separate phenomenon from other pathology, requiring that the NSSI be addressed first in order to prevent escalation of the behavior. In addition, both approaches suggest the conducting a thorough behavior analysis of the NSSI with the goal of increas- 
ing understanding, reducing the positive and negative reinforcement of the behavior, and identifying where skill deficits interfere with adaptive coping. Last of all, both PST and DBT are flexible enough to accommodate the integration of other cognitive-behavior skills so that the therapist can match the treatment to the client's skills deficits, fostering a collaborative atmosphere.

Although research is lacking in regard to the effective elements of these therapies in reducing NSSI, a number of researchers have speculated on what the active mechanisms of change may be. One element identified is the functional analysis of the NSSI (Hopko et al., 2003; Verheul et al., 2003) because this provides a context for the NSSI along with the potential to identify skill deficits. The identification and remediation of skill deficits, particularly emotion regulation sills, is also viewed as a key component for reducing NSSI. The teaching of specific skills is critical because it assists the client in developing an adaptive capacity to interact with the world, tolerate distress, and regulate emotions (Robins \& Chapman, 2004). Some of the primary skill deficits include but are not limited to problem-solving (generating alternative solutions in particular), distress tolerance, interpersonal communication, and coping strategies. Related to providing specific skill sets, maintaining an explicit focus on behavioral modification and rehearsal is also important to ensuring skill acquisition and generalization to daily life (Verheul et al., 2003). Therefore, therapy should include role plays and in-session practice to increase the client's self-efficacy in using the newly acquired skills. The last element often speculated to contribute to effective change is the modification of cognitive distortions and negative core beliefs. Underlying beliefs about the nature of NSSI, the self, and how to cope with negative emotion states can play a critical role in initiating or maintaining NSSI (Walsh \& Rosen, 1988). Of the research reviewed, the studies that demonstrated success in reducing NSSI included some element of cognitive restructuring, suggesting that this is a critical piece. To summarize, the mechanisms of change for effectively treating NSSI are largely unknown and purely speculative. However, it seems as though a comprehensive, multi-dimensional approach that incorporates basic techniques from behavioral, cognitive, and problem-solving treatments will likely produce the greatest effect.

\section{GENERAL TREATMENT RECOMMENDATIONS}

Drawing upon the literature reviewed and hypothesized mechanisms of change, there appears to be a consensus of general treatment recommendations to guide mental health counselors who are working with clients who engage in NSSI. At this time, the treatment elements can be broken 
down into four general categories: therapeutic relationship, functional behavioral analysis, behavioral interventions, and cognitive restructuring. Each of these are discussed below.

\section{Therapeutic Relationship and Alliance}

It is important to acknowledge that there are few resources discussing elements of therapy thought to be critical to reducing non-suicidal selfinjury (NSSI). Some additional guidance can be obtained from the empirical and theoretical resources highlighting recommendations for working with suicidal individuals (e.g., Henriques, Beck, \& Brown, 2003; Jobes, 2000; Rudd, Joiner, Jobes, \& King, 1999). From this literature, it is clear that forming a supportive and collaborative therapeutic alliance where the client and therapist work as a team rather than "expert" and "subject" is paramount to successfully treating self-harming behaviors (Jobes \& Drozd, 2004; Orbach, 2001; Sidley, 1998). When working with individuals who engage in NSSI and are not imminently suicidal, forming an especially strong working alliance is seen as an essential first step to actively targeting the behavior because the relationship is often challenged and can also become part of the intervention strategies used (Hawton, 1990; Linehan, 1993).

One way to establish such a relationship is to acknowledge the extent of pain the client is likely to be in along with the functional component the NSSI is providing. Orbach (2001) explains that being able to understand and acknowledge the client's pain, as well as why the NSSI appears to be a viable option is key to establishing a strong alliance because it communicates a willingness by the therapist to join the client where she or he is at without fear. It is also useful to view the NSSI as an expression of emotion and as an effective, albeit self-destructive, coping tool for the client because this will promote adoption an empathic stance (Collins, 1996; Orbach, 2001). Helping the client to articulate the perceived adaptive coping elements could further facilitate a feeling of being understood and make discussions of the less adaptive functions more amenable to the client (Linehan, 1993). Once a strong therapeutic alliance is formed, the next goal of therapy is to reduce and ultimately eliminate the NSSI, replacing it with more adaptive coping skills. As stressed in the literature, the primary focus of treatment should be the NSSI, and only after it is under control should therapy specifically focus on the underlying pathology (Boyce, Oakley-Browne, \& Hatcher, 2001; Linehan, 2000). One may see a reduction in Axis I pathology by targeting the NSSI since similar problem areas can underlie both dysfunctions. 


\section{Functional Behavioral Analysis}

One of the first steps toward reducing NSSI is to conduct an extensive behavioral analysis of each incident, or the identified key incident in the case of multiple acts (Favazza, 1996; Kernberg, 1994; Linehan, 1993, 2000). It is crucial that the client and therapist work together to identify the precipitating and maintaining events associated with the NSSI acts (Favell et al., 1982; Hawton, 1990; Linehan, 1993), including cognitive, emotional, and environmental factors. Conducting a behavioral analysis provides the therapist and client with an understanding of the context surrounding the NSSI. This is important for identifying skill deficits that can inform interventions and generate alternative coping strategies to prevent future NSSI. Identifying thoughts, emotions, and associated behaviors that increase or decrease the likelihood of self-injury as part of the behavioral analysis is important since many acts of NSSI are precipitated by aversive internal states. Identifying maladaptive emotions, cognitions, or behaviors that contribute to the NSSI provide cues of where to direct specific interventions strategies and what type of intervention may produce the greatest effect. For example, if a client repeatedly identifies thoughts of deserving punishment as precipitating the NSSI cognitive restructuring of this thought may be warranted. On the other hand, if the client identifies intense, overwhelming anger as a precipitant the best intervention may be anger management skills. The functional behavioral analysis provides a roadmap that can directly inform the therapist's intervention strategies.

\section{Behavioral Interventions}

After the elements or patterns associated with the NSSI are identified, therapy should focus on the behavioral aspects of the behavior if indicated by the functional analysis. Behavioral interventions that work on identifying and eliminating the positive and negative reinforcements of the NSSI will likely produce the greatest effect. This is accomplished by helping the client to discover alternative ways to express emotions, by rewarding reductions in the frequency, severity, or type of self-injury, and by encouraging others to react in a neutral, yet supportive, fashion (Favell et. al., 1982; Van Moffaert, 1990; Zila \& Kiselica, 2001). Involving others from the client's environment such as parents, friends, or partners may also help to reduce external reinforcers. Helping the client to identify aversive short- and long-term consequences associated with the NSSI should also be used to increase sensitivity to potential extinguishing factors.

In addition to addressing positive and negative reinforcements of NSSI, behavioral strategies can include the teaching of new skills. Training in 
specific coping skills or distress tolerance can address behavioral deficits in managing psychological or emotional anguish. Generating alternative behaviors that the client can engage in instead of the NSSI, and shaping the use of such behaviors is a solid behavioral method to employ. Therapy can also address potential deficits in problem-solving, communication skills, and the identification, labeling, and verbalization of emotions (Bowen \& John, 2001; Collins, 1996; Hawton, 1990), all of which are areas that self-injuring individuals have shown deficits. Non-suicidal self-injury is often used to escape uncomfortable affective or interpersonal states. Desensitization techniques can be used to increase the client's ability to withstand intense emotions and interpersonally aversive experiences both within and outside of session (Linehan, 1993; Walsh \& Rosen, 1988). Training in intimacy, conflict resolution, and adaptive coping are also highly recommended since self-injuring individuals tend to have weak skills in these areas as well (Evans, 2000; Hawton, 1990; Kernberg, 1994).

\section{Cognitive Restructuring}

In conjunction with the behavioral interventions, cognitive restructuring of maladaptive beliefs will likely be necessary (Zila \& Kiselica, 2001). Individuals who engage in NSSI often have a number of negative automatic thoughts about themselves or others, their body, their ability to cope with distress, and their future (Favazza, 1996). Along with these more global cognitive distortions, Walsh and Rosen (1988) highlight four key cognitions common to people who engage in NSSI that are likely to initiate and perpetuate the self-injury. First, the individuals often believe that self-injury is acceptable or necessary. Second, they believe that one's body and self are disgusting and deserving of punishment, making it acceptable for the body to be the target of self-abuse. Underlying this belief are thoughts of self-loathing, that one deserves to be punished, or that one needs to harm the "bad" self. A third belief is that action is needed to reduce unpleasant feelings or to solve the immediate crisis. This belief can serve as the initiator for NSSI when one is experiencing unbearable distress. Finally, there is the belief that overt action is needed to communicate feelings to others and to have others fully understand the extent of the suffering.

Utilizing cognitive therapy techniques to challenge and change these key beliefs will likely result in a reduction and potentially a cessation of the NSSI. It is important to develop and strengthen the idea that selfinjury is incompatible with self-respect and self-esteem so that as selfworth increases the NSSI becomes less viable as a coping method. In addition, therapy should modify the other dysfunctional beliefs and neg- 
ative automatic thoughts the client may have that contribute to the NSSI (e.g., being unlovable), replacing them with more adaptive thoughts through standard cognitive therapy.

\section{CONCLUDING COMMENTS AND FUTURE DIRECTIONS}

Although limited in their conclusions, the existing studies evaluating the efficacy of cognitive-behavioral interventions for NSSI behavior provide promising information regarding treatment. There is some concern among professionals that the incidence of NSSI is rising and that more people are going to be seeking treatment for self-injury. This forecast requires mental health professionals and researchers to be ready to treat such difficult behaviors. Self-injurious behaviors are complex and encompass a variety of additional comorbid psychological problems, and this poses a number of challenges. Mental health professionals must be ready to respond with creative, innovative, and effective treatments. Consequently, it is imperative that mental health counselors and researchers begin to conduct controlled clinical trials on the efficacy of different treatments for NSSI as well as identify what components of a treatment appear to be most efficacious. One additional concern is that many improvements seen at the end of therapy were not maintained over time relative to treatment as usual. This lack of long-term effectiveness may be a result of interventions that were too short, a failure of skills generalization, or lack of power in statistical analyses to detect effects. Research that examines potential mediating and moderating variables contributing to or preventing long-term success is greatly needed, as is a study incorporating what seem to be the effective elements into one treatment condition compared to DBT, PST, or treatment as usual. Based upon the research reviewed, it is recommended that cognitive-behavioral problem-solving therapies and dialectical behavior therapy be rigorously studied because these two approaches have demonstrated the greatest potential to date for being effective.

As a concluding note, it is important to recognize some of the challenges that treatments for NSSI will have to overcome. Probably the most relevant challenge is that individuals who engage in NSSI are highly heterogeneous, so creating a standardized treatment that is effective for all will be difficult. Therapies should be multi-modal and have standardized interventions shown to be effective in treating specific aspects of the disorder (e.g., maladaptive cognitions, problem-solving skills, distress tolerance), yet still be flexible enough to be tailored to the individual needs of the client. Forming a therapeutic alliance may also be a challenge since many individuals who engage in NSSI acts have often experienced inter- 
personal loss and rejection, and have difficulty forming trusted intimate relationships. Creating a therapeutic alliance may provide a significant corrective experience for the client, and may be an effective intervention on its own, so it is important that a strong alliance be developed. Likewise, the alliance can be used at times to provide social reinforcement for reductions in parasuicide. Finally, due to the powerful reinforcements that coincide with NSSI, finding adaptive replacement behaviors for dealing with aversive experiences that provide similar relief for the client is challenging. Creativity, flexibility, and perseverance on the part of the clinician are paramount.

\section{REFERENCES}

Barley, W.D., Buie, S.E., Peterson, E.W., Hollingsworth, A.S., Griva, M., Hickerson, S.C., et al. (1993). Development of an inpatient cognitive-behavioral treatment program for borderline personality disorder. Journal of Personality Disorders, 7, 232-240.

Bauserman, S. A. K. (1998). Treatment of persons who self-mutilate with dialectical behavior therapy. Psychiatric Rehabilitation Skills, 2, 149-157.

Bohus, M., Haaf, B., Simms, T., Limberger, M.F., Schmahl, C., Unckel, C., et al. (2004). Effectiveness of inpatient dialectical behavior therapy for borderline personality disorder: A controlled trial. Behaviour Research and Therapy, 42, 487-499.

Bohus, M., Haaf, B., Stiglmayr, C., Phol, U., Boehme, R., \& Linehan, M. (2000). Evaluation of inpatient dialectical behavior therapy for borderline personality disorder: A prospective study. Behaviour Research and Therapy, 38, 878-887.

Bowen, A. C. L., \& John, A. M. H. (2001). Gender differences in presentation and conceptualization of adolescent self-injurious behaviour: Implications for therapeutic practice. Counselling Psychology Quarterly, 14, 357-379.

Boyce, P., Oakley-Browne, M. A., \& Hatcher, S. (2001). The problem of deliberate self-harm. Current Opinion in Psychiatry, 14, 107-111.

Briere, J. \& Gil, E. (1998). Self-mutilation in clinical and general population samples: Prevalence, correlates, and functions. American Journal of Orthopsychiatry, 68, 609-620.

Collins, D. (1996). Attacks on the body: How can we understand self-harm? Psychodynamic Counselling, 2, 463-475.

Crowe, M., \& Bunclark, J. (2000). Repeated self-injury and its management. International Review of Psychiatry, 12, 48-54.

Derouin, A., \& Bravender, T. (2004). Living on the edge: The current phenomenon of selfmutilation in adolescents. The American Journal of Maternal/Child Nursing, 29, 12-18.

D'Zurilla, T. J., \& Goldfried, M. R. (1971). Problem solving and behavior modification. Journal of Abnormal Psychology, 78, 107-126.

D'Zurilla, T. J. \& Nezu, A. M. (2001). Problem solving therapies. In K. Dobson (Ed.) Handbook of Cognitive-Behavioral Therapies (2nd Edition) (pp. 211-245). New York: Guilford Press.

Elwood, L. M., Comtois, K. A., Holdcraft, L. C., \& Simpson, T. L. (2002, November). Effectiveness of a dialectical behavior therapy in a community mental health center. Paper presented at the annual meeting of the Association for Advancement of Behavior Therapy, Reno, NV. 
Evans, J. (2000). Interventions to reduce repetition of deliberate self-harm. International Review of Psychiatry, 12, 44-47.

Evans, K., Tyrer, P., Catalan, J., Schmidt, U., Davidson, K., Dent, J. et al., (1999). Manualassisted cognitive-behaviour therapy (MACT): A randomized controlled trial of a brief intervention with bibliotherapy in the treatment of recurrent deliberate self-harm. Psychological Medicine, 29, 19-25.

Evans, J., Williams, J. M., O’Loughlin, S., \& Howells, K. (1992). Autobiographical memory and problem-solving strategies of parasuicidal patients. Psychological Medicine, 22, 399-405.

Favazza, A. R. (1996). Bodies Under Siege: Self-Mutilation and Body Modification in Culture and Psychiatry (2nd Ed.). Baltimore, MD: John Hopkins University Press.

Favazza, A. R. (1998). The coming of age of self-mutilation. The Journal of Nervous and Mental Disease, 186, 259-268.

Favell, J. E., Azrin, N. H., Baumeister, A. A., Carr, E. G., Dorsey, M. F., Forehand, R., et al. (1982). The treatment of self-injurious behavior. Behavior Therapy, 13, 529-554.

Gibbons, J. S., Butler, J., Urwin, P., \& Gibbons, J. L. (1978). Evaluation of a social work service for self-poisoning patients. British Journal of Psychiatry, 133, 111-118.

Gratz, K. L. (2001). Measurement of deliberate self-harm: Preliminary data on the deliberate self-harm inventory. Journal of Psychopathology and Behavioral Assessment, 23, 253-263.

Hawton, K. (1990). Self-cutting: Can it be prevented? In K. Hawton and P.Cowen (Eds.) Dilemmas and Difficulties in the Management of Psychiatric Patients (pp. 91-103). New York: Oxford University Press.

Hawton, K., Arensman, E., Townsend, E., Bremner, S., Feldman, E., Goldney, R., et al., (1998). Deliberate self-harm: Systematic review of efficacy of psychosocial and pharmacological treatments in preventing repetition. British Medical Journal, 317, 441- 447.

Hawton, K., Fagg, J., Simkin, S., Bale, E., \& Bond, A. (1997). Trends in deliberate self-harm in Oxford. 1985-1995. British Journal of Psychiatry, 171, 556-560.

Hawton, K., McKeown, S., Day, A., Martin, P., O'Conner, M., Yule, J., et al. (1987). Evaluation of out-patient counseling compared with general practitioner care following overdoses. Psychological Medicine, 17, 751-761.

Henriques, G., Beck, A. T., \& Brown, G. K., (2003). Cognitive therapy for adolescent and young adult suicide attempters. American Behavioral Scientist, 46, 1228-1268.

Hopko, D.R., Sanchez, L., Hopko, S.D., Dvir, S., \& Lejuez, C.W. (2003). Behavioral activation and the prevention of suicidal behaviors in patients with borderline personality disorder. Journal of Personality Disorders, 17, 460-465.

Ivanhoff, A., Linehan, M. M., \& Brown, M. (2001). Dialectical behavior therapy for impulsive self-injurious behaviors. In D. Simeon and E. Hollander (Eds.) Self-Injurious Behaviors: Assessment and Treatment (pp. 149-173). Washington, D.C.: American Psychiatric Publishing, Inc.

Jobes, D. A. (2000). Collaborating to prevent suicide: A clinical-research perspective. Suicide and Life-Threatening Behavior, 30, 8-17.

Jobes, D.A., \& Drozd, J. F. (2004). The CAMS approach to working with suicidal patients. Journal of Contemporary Psychotherapy, 34, 73-85.

Katz, L.Y., Cox, B.J., Gunasekara, S., \& Miller, A. L. (2004). Feasibility of dialectical behavior therapy for suicidal adolescent inpatients. Journal of the American Academy of Child \& Adolescent Psychiatry, 43, 276-282.

Kernberg, P. F. (1994). Psychological interventions for the suicidal adolescent. American Journal of Psychotherapy, 48, 52-63. 
Koons, C., Robins, C. J., Tweed, J. L., Lynch, T. R., Gonzalez, A. M., Morese, J. Q., et al. (2001). Efficacy of dialectical behavior therapy in women veterans with borderline

personality disorder. Behavior Therapy, 32, 371-390.

Lerner, M. S., \& Clum, G. A. (1990). Treatment of suicide ideators: A problem-solving approach. Behavior Therapy, 21, 403-411.

Liberman, R. P., \& Eckman, T. (1981). Behavior therapy vs. insight-oriented therapy for repeated suicide attempters. Archives of General Psychiatry, 38, 1126-1130.

Linehan, M. M. (1993). Cognitive-Behavioral Treatment of Borderline Personality Disorder. New York: Guilford Press.

Linehan, M. M. (2000). Behavioral treatments of suicidal behaviors: Definitional obfuscation and treatment outcomes. In R. W. Maris, S. S. Cannetto, J. L. McIntosh, \& M. M. Siverman (Eds.), Review of Suicidology, (pp. 84-111). New York, NY: Guilford Press.

Linehan, M. M., Armstrong, H. E., Suarez, A., Allmon, D., \& Heard, H. L. (1991). Cognitive behavioral treatment of chronically parasuicidal borderline patients. Archives of General Psychiatry, 48, 1060-1064.

Linehan, M. M., Comtois, K. A., Brown, M. Z., Reynolds, S. K., Welch, S. S., Sayrs, J., \& Korslund, K. E. (2002, November). DBT vs. non-behavioral treatment by experts in the community: Clinical outcomes at one year. In S. K. Reynolds (Chair), The University of Washington study for borderline personality disorder: DBT vs. nonbehavioral treatment by experts in the community. Symposium conducted at the annual meeting of the Association for Advancement of Behavior Therapy, Reno, NV.

Linehan, M. M., Dimeff, L. A., Reynolds, S. K., Comtois, K. A., Welch, S. S., Heagerty, P., \& Kivlahan, D. R. (2002). Dialectical behavior therapy versus comprehensive validation therapy plus 12-step for the treatment of opioid dependent women meeting criteria for borderline personality disorder. Drug and Alcohol Dependence, 67, 13-26.

Linehan, M. M., Heard, H. L., Armstrong, H. E. (1993). Naturalistic follow-up of a behavioral treatment for chronically parasuicidal borderline patients. Archives of General Psychiatry, 50, 971-974.

Low, G., Jones, D., Duggan, C., Power, M., \& MacLeod, A. (2001). The treatment of deliberate self-harm in borderline personality disorder using dialectical behavior therapy: A pilot study in a high security hospital. Behavioural \& Cognitive Psychotherapy, 29, 85-92.

MacLeod, A. K., Williams, J. M. G., \& Linehan, M. M. (1992). New developments in the understanding and treatment of suicidal behavior. Behavioural Psychotherapy, 20, 193-218.

McLeavey, B. C., Daly, R. J., Ludgate, J. W., \& Murray, C. M. (1994). Interpersonal problemsolving skills training in the treatment of self-poisoning patients. Suicide \& LifeThreatening Behavior, 24, 382-394.

Miller, A L. (1999). Dialectical behavior therapy: A new treatment approach for suicidal adolescents. American Journal of Psychotherapy, 53, 413-417.

Miller, A. L., \& Glinski, J. (2000). Youth suicidal behavior: Assessment and intervention. Journal of Clinical Psychology, 56, 1131-1152.

Miller, A. L., Wyman, S. E., Huppert, J. D., Glassman, S. L., \& Rathus, J. H. (2000). Analysis of behavioral skills utilized by suicidal adolescents receiving dialectical behavior therapy. Cognitive \& Behavioral Practice, 7, 183-187.

Nock, M. K., \& Prinstein, M. J. (2004). A functional approach to the assessment of selfmutilative behavior. Journal of Consulting and Clinical Psychology, 72, 885-890.

Orbach, I. (2001). Therapeutic empathy with the suicidal wish: Principles of therapy with suicidal individuals. American Journal of Psychotherapy, 55, 166-185.

Patsiokas, A. T., \& Clum, G. A. Effects of psychotherapeutic strategies in the treatment of suicide attempters. Psychotherapy, 22, 281-290. 
Perseius, K.I., Ojehagen, A., Ekdahl, S., Asberg, M., \& Samuelsson, M. (2003). Treatment of suicidal and deliberate self-harming patients with borderline personality disorder using dialectical behavior therapy: The patients' and the therapists' perceptions. Archives of Psychiatric Nursing, 17, 218-227.

Pollock, L. R., \& Williams, J.M.G. (1998). Problem solving and suicidal behavior. Suicide and Life-Threatening Behavior, 28, 375-387.

Raj, A. J., Kumaraiah, V., \& Bhide, A. V. (2001). Cognitive-behavioral intervention in deliberate self-harm. Acta Psychiatrica Scandinavica, 104, 340-345.

Rathus, J. H., \& Miller, A. L. (2002). Dialectical behavior therapy adapted for suicidal adolescents. Suicide and Life-Threatening Behavior, 32, 146-157.

Robins, C. J., \& Chapman, A. L. (2004). Dialectical behavior therapy: Current status, recent developments, and future directions. Journal of Personality Disorders, 18, 73-89.

Rudd, M.D., Joiner, T.E., Jobes, D.A., \& King, C.A. (1999). Practice guidelines in the outpatient treatment of suicidality: An integration of science and a recognition of its limitations. Professional Psychology: Research and Practice, 30, 437-446.

Rudd, M. D., Rajab, M. H., Orman, D. T., Joiner, T., Stulman, D. A., \& Dixon, W. (1996). Effectiveness of an outpatient intervention targeting suicidal young adults: Preliminary results. Journal of Consulting and Clinical Psychology, 64, 179-190.

Salkovskis, P. M., Atha, C., \& Storer, D. (1990). Cognitive-behavioural problem-solving in the treatment of patients who repeatedly attempt suicide: A controlled trial. British Journal of Psychiatry, 157, 871-876.

Scheel, K. R. (2000). The empirical basis of dialectical behavior therapy: Summary, critique, and implications. Clinical Psychology: Science and Practice, 7, 68-86.

Shearin, E. N., \& Linehan, M. M. (1992). Patient-therapist ratings and relationship to progress in dialectical behavior therapy for borderline personality disorder. Behavior Therapy, 23, 730-741.

Shearin, E. N., \& Linehan, M. M. (1994). Dialectical behavior therapy for borderline personality disorder:Theoretical and empirical foundations. Acta Psychiatrica Scandinavica, $89,61-68$.

Sidley, G. L. (1998). Parasuicide. In N. Tarrier, A. Wells, and G. Haddock (Eds.) Treating Complex Cases: The Cognitive Behavioural Therapy Approach (pp. 272 -293). New York: John Wiley \& Sons Ltd.

Simpson, E. B., Pistorello, J., Begin, A., Costello, E., Levinson, J., Mulberry, S., et al. (1998). Use of dialectical behavior therapy in a partial hospital program for women with borderline personality disorder. Psychiatric Services, 49, 669-673.

Speckens, A.E.M., \& Hawton, K. (2005). Social problem solving in adolescents with suicidal behavior: A systematic review. Suicide and Life-Threatening Behavior, 35, 365-387.

Springer, T., Lohr, N.E., Buchtel, H.A., \& Silk, K.R. (1996). A preliminary of short-term cognitive-behavioral group therapy for inpatients with personality disorders. Journal of Psychotherapy Practice and Research, 5, 57-71.

Townsend, E., Hawton, K., Altman, D. G., Arensman, E., Gunnell, D., Hazell, P., et al., (2001). The efficacy of problem-solving treatments after deliberate self-harm: Meta-analysis of randomized controlled trials with respect to depression, hopelessness, and improvement in problems. Psychological Medicine, 31, 979-988.

Tryer, P., Thompson, S., Schmidt, U., Jones, V., et al. (2003). Randomized controlled trial of brief cognitive behaviour therapy versus treatment as usual in recurrent deliberate selfharm: the POMPACT study. Psychological Medicine, 33, 969-976. 
Tryer, P., Tom, B., Byford, S., Schmidt, U., Jones, V., Davidson, K., et al. (2004). Differential effects of manual assisted cognitive behaviour therapy (MACT) in the treatment of recurrent deliberate self-harm and personality disturbance: The POMPACT study. Journal of Personality Disorders, 18, 102-116.

Turner, R. M. (2000). Naturalistic evaluation of dialectical behavior therapy-oriented treatment for borderline personality disorder. Cognitive and Behavioral Practice, 7, 413-419.

Van Moffaert, M. M. P. (1990). Self-mutilation: Diagnosis and practical treatment. International Journal of Psychiatry in Medicine, 20, 373-382.

Verheul, R., van den Bosch, L. M., Koeter, M. W. J., deRidder, M. A. J., Stijnen, T., \& van den Brink, W. (2003). Dialectical behavior therapy for women with borderline personality disorder. British Journal of Psychiatry, 182, 135-140.

Walsh, B. W., \& Rosen, P. M. (1988). Self-Mutilation: Theory, Research, and Treatment. New York: Guilford Press.

Zila, L. M., \& Kiselica, M. S. (2001). Understanding and counseling self-mutilation in female adolescents and young adults. Journal of Counseling and Development, 79, 46-53. 
Copyright of Journal of Mental Health Counseling is the property of American Mental Health Counselors Association and its content may not be copied or emailed to multiple sites or posted to a listserv without the copyright holder's express written permission. However, users may print. download, or email articles for individual use. 